\title{
Opacity Problems in Protostellar Objects
}

\author{
Harold W. Yorke ${ }^{1}$ and Thomas Henning ${ }^{2}$ *
}

1 Institut für Astronomie und Astrophysik, Am Hubland, D-97074 Würzburg, Germany

2 Max Planck Society, Research Group: "Dust in Star-Forming Regions", Schillergäßchen 2-3, D-07745 Jena, Germany

\section{Introduction}

Opacity influences the transfer of radiation and is thus of decisive importance for (a) the interpretation of astronomical observations, (b) determining the thermal structure, (c) critically influencing molecular, atomic and thermonuclear processes and (d) affecting the overall dynamic evolution during the star formation process. Questions pertaining to the onset of instability of gas clouds, their subsequent fragmentation into smaller clumps, the formation of multiple systems, the initial mass function (IMF), the upper and lower mass limits, star formation efficiency and the fate of material not collected into stars cannot be considered without intimate knowledge of the opacity in this environment.

It is important to note that, when present, dust dominates the opacity by several orders of magnitude in comparison to molecular or atomic contributions (see e.g. Yorke 1985). Dust is absent in the star/protostar and its immediate vicinity, where the radiation field is too intense, i.e. where the equilibrium grain temperature exceeds the sublimation temperature or chemical sputtering takes place. This and a multitude of other processes (to be discussed in section 2) alter the mixture of grain types, sizes and shapes which can exist at different parts within the protostellar envelope.

We introduce in this context the concept of XNLTE. Whereas in Local Thermodynamic Equilibrium (LTE) the opacity can be unambiguously determined by local conditions, say the chemical composition $Z_{i}$ and two state variables, density $\rho$ and temperature $T$, there is a spatial interconnectivity associated with NLTE. In addition to local conditions the opacity depends on the radiation field. Usually it suffices to specify the $0^{t h}$ moment $J_{\nu}$ of the radiation intensity at all frequencies $\nu$. In the case of XNLTE (eXtreme Non-Local Thermodynamic Equilibrium) there is also a temporal interconnectivity. The opacity depends not only on the global state of the medium but on its overall evolutionary history. This is particularly true for dust opacities in the stellar/protostellar environment.

\footnotetext{
* This work was supported by the DFG (German research Foundation). Numerical calculations were conducted on the CRAY Y-MPs at the HLRZ (Jülich) and at the LRZ (Munich) computing centers.
} 


$$
\begin{array}{ll}
\text { LTE: } & \kappa_{\nu}=\kappa_{\nu}\left(\rho, T, Z_{i}\right) \\
\text { NLTE: } & \kappa_{\nu}=\kappa_{\nu}\left(\rho, T, Z_{i} ; J_{\nu^{\prime}}\right) \\
\text { XNLTE: } & \kappa_{\nu}=\kappa_{\nu}\left(\rho, T, Z_{i} ; J_{\nu^{\prime}} ; \text { history }\right)
\end{array}
$$

\subsection{Basic types of dust mixtures}

We shall distinguish between four basic types of interstellar dust mixtures and include for completion a fifth type, interplanetary dust (see Figure 1).

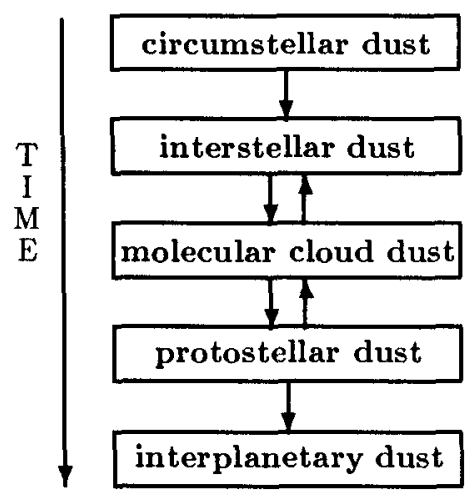

Fig. 1. Evolutionary sequence from circumstellar to interplanetary dust

Circumstellar dust. Dust forms in the outer regions of cool stars (see e.g. Sedlmayr this volume), in novae and in supernovae (see e.g. Evans 1990, Kozasa et al. 1991). The composition and size distribution which forms and evolves in this environment depends on the chemical abundances, the density, temperature and velocity structure and thus can be expected to vary from object to object.

Diffuse interstellar dust. In the general interstellar medium $\left(n_{\mathrm{H}} \lesssim 10 \mathrm{~cm}^{-3}\right)$ grains from various sources have intermixed. Because of frequent reprocessing due to the passage of shocks, adsorption ${ }^{1}$ of gaseous species, the effect of the diffuse interstellar UV field, cosmic rays and having spent several previous periods of time in the molecular cloud phase, the dust has developed into a homogeneous sample. Its overall optical properties appear to be constant within the Galaxy and similar to that observed in the interstellar medium of other galaxies. A "classical" model for the size distribution assumes a power law number density of spherical dust grains $n(a) \propto a^{-3.5} d a$ for grain radii in the range $50 \AA \leq \mathrm{a} \leq 0.25 \mu \mathrm{m}$ (MRN distribution, Mathis et al. 1977). Current grain models include a fraction of very small grains (VSG) with a radius of about $10 \AA$.

${ }^{1}$ adsorption $=$ process of taking up molecules and atoms onto the surface of a solid or liquid and binding them by van der Waals forces, ionic or chemical bonds 
Molecular cloud dust. Due to the comparatively high densities in molecular clouds $\left(n_{\mathrm{H}_{2}} \gtrsim 10^{3} \mathrm{~cm}^{-3}\right)$ the adsorption of gaseous species proceeds at a faster rate, grain surface chemistry becomes more important than in the diffuse interstellar state and the grains develop dirty "icy" mantels. Theoretically, one can expect a mixture of $\mathrm{H}_{2} \mathrm{O}, \mathrm{CH}_{3} \mathrm{OH}, \mathrm{CO}$ and some other simple molecules (see e.g. Whittet 1993). The exact composition is very uncertain and, since it reflects the physical conditions and history of the grain's environment, should vary from cloud to cloud. Embedded in the icy coatings one can expect to find impurities such as amorphous carbon particles (see e.g. Preibisch et al. 1993). Effects of grain coagulation will modify the size distribution and lead to larger composite and fluffy grains (Ossenkopf 1993). Depending on particle composition van-der-Waals bonds or H-bridges determine the sticking coefficient. High relative velocities between colliding grains, say $v_{\text {rel }} \geq 10^{4} \mathrm{~cm} \mathrm{~s}^{-1}$, may also occur and will generally be destructive, so that the overall problem of grain growth cannot be expected to be straight-forward.

Protostellar dust. In the protostellar environment densities $n_{\mathrm{H}_{2}} \geq 10^{8} \mathrm{~cm}^{-3}$ occur in the disks formed around the central hydrostatic object (which we here designate "protostar"). Processes as described above for molecular clouds are prevalent but at a much higher rate. We shall elaborate on this in the following sections.

Interplanetary dust. Interplanetary dust is the result of the extensive preprocessing taking place in the protostellar environment. Adsorption, accretion, coagulation and gravitational agglomeration, in addition to further chemical evolution and thermal annealing (repeated heating and cooling), occurs to the extent that macroscopic particles are formed. These processes, combined with the effects of destructive collisions and the destruction of the outer icy layers of comets, lead to the characteristic types of interplanetary dust observed in our Solar System. Since a detailed discussion of interplanetary dust is beyond the scope of the present work, we will not consider it further here and instead refer the interested reader to recent reviews (see e.g. Mukai 1990, Levasseur-Regourd and Hasegawa 1991).

\subsection{Dust Models}

When developing a new dust model the following steps are generally followed:

1. Assume (or attempt to calculate ab initio) chemical composition, shape and size of each grain type.

2. Use "measured" dielectric properties. This is not necessarily trivial, because the dielectric properties are in general not known for all materials and for all wavelengths of astrophysical interest. Also, the interpretation of laboratory measurements is often model dependent. 

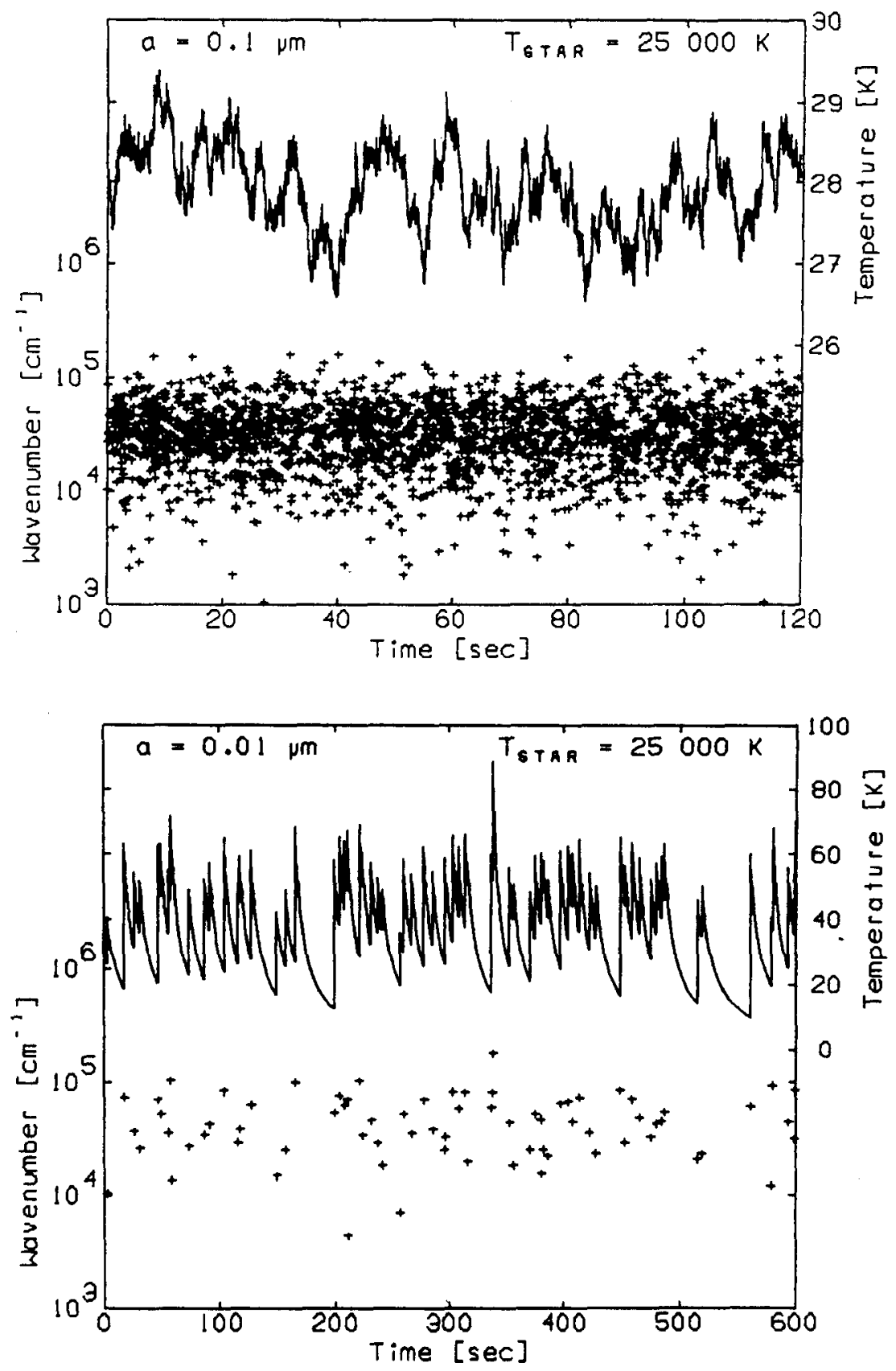

Fig. 2. Monte-Carlo simulation of the evolution of grain temperature (solid lines) for a dust particles of radius $a=10^{-5} \mathrm{~cm}$ (top panel) and $a=10^{-6} \mathrm{~cm}$ (bottom panel) located $0.3 \mathrm{pc}$ from a B0 star. The wavenumber and arrival time for each individual photon is indicated by a ' + ' (see Yorke 1988 for details). 
3. Calculate the optical properties of each grain type using e.g.

- Mie theory (spheres), theories for rotationally symmetric particles (spheroids, finite cylinders)

- interaction of light with small particles (lattice vibrations, surface modes, luminescence, ...)

- new methods for irregular, anisotropic fluffy, fractal grains (discrete dipole approximation, effective medium theories combined with T-matrix method or scattering matrix method)

4. Construct appropriate mean values. Again, this is not always trivial. For instance, can one use the mean properties of a mixture of grains of differing sizes? Or should each size be treated as a separate component (see Figure 2)? Is one allowed to average over different compositions? Generally speaking, each averaging assumption should be carefully tested with respect to its extent of validity.

5. Work with the model. And compare to observations.

The common features of all current dust models (see Table 1) are (1) they contain some form of silicate and carbonaceous material and (2) they all claim to be able to fit the observed extinction and polarization observations. The first item can be interpreted as a general concensus that indeed carbon and silicates are important constituents of the dust, whereas the second item is indicative of the non-uniqueness of the model predictions. The basic differences in the models are the assumed size and shape distributions, the possible existence of organic mantles in the diffuse interstellar medium (ISM) and the origin of certain characteristic features, such as the near IR emission features and the $220 \mathrm{~nm}$ ultraviolet absorption bump. We summarize the basic characteristics of some current grain models in Table 1 (updated and adapted from Mathis 1990).

Problems with current dust models are: the lack of good optical constants of the individual components over a broad wavelength region, our poor understanding of the influence of grain shape and porosity, our lack of detailed understanding of the observed polarization, and, of course, the non-uniqueness of the models. To a certain extent, these shortcomings can be overlooked, i.e. when one is only interested in the global effects of opacity on the spectral appearance. Often, however, the details of the dust model critically influence the evolution.

\section{Evolution of Protostellar Objects}

We are accustomed to viewing the formation and early evolution of stars as a four step process (see e.g. Shu et al. 1987):

a.) Formation of dense molecular cores and cloud fragments which presumably are supported by magnetic fields.

b.) After the onset of gravitational instability a fragment with non-zero angular momentum collapses and forms a central hydrostatic core $\left(M \approx 10^{-3} \mathrm{M}_{\odot}\right.$, i.e. the protostar) surrounded by a disk. Both protostar and disk grow in mass as they accrete matter from the molecular cloud fragment. 
Table 1. Characteristics of some current grain models

\begin{tabular}{|c|c|c|c|c|}
\hline $\begin{array}{l}\text { Brief title of theory } \\
\text { (and authors) }\end{array}$ & Composition of grains & $\begin{array}{l}\text { Size distr. } \\
\text { grains }\end{array}$ & $\begin{array}{l}\text { Carrier of } \\
\text { bump }\end{array}$ & Refs. \\
\hline $\begin{array}{l}\text { Core/mantle } \\
\text { (Greenberg) }\end{array}$ & $\begin{array}{l}\text { Silicate cores/organic } \\
\text { refractory mantles; also } \\
\text { small silicates, graphite }\end{array}$ & "Flat"b & $\begin{array}{l}\text { Small } \\
\text { graphite }^{c}\end{array}$ & 1,2 \\
\hline $\begin{array}{l}\text { Core-mantle + iron } \\
\text { (Chlewicki, Laureijs) }\end{array}$ & $\begin{array}{l}\text { Similar as Greenberg, with } \\
\text { small metallic iron particles, } \\
\text { PAHs }\end{array}$ & "Flat"b & $\begin{array}{l}\text { Small } \\
\text { graphite }^{c}\end{array}$ & 3 \\
\hline Draine-Lee (or MRN) & Bare silicate, graphite & $\mathrm{a}^{-3.5}$ & Graphite & 4,5 \\
\hline Duley/Jones/Williams & $\begin{array}{l}\text { Silicate cores, hydrogenated } \\
\text { amor. C clusters, small sili- } \\
\text { cates }\end{array}$ & $a^{-3.5}$ & $\begin{array}{l}\text { OH-ions } \\
\text { near Si }\end{array}$ & 6 \\
\hline $\begin{array}{l}\text { Composite grains } \\
\text { (Mathis, Whiffen) }\end{array}$ & $\begin{array}{l}\text { Silicates, amor. C, small } \\
\text { graphite in the same grains } \\
\text { (with free small graphite, } \\
\text { possibly silicates) }\end{array}$ & $\mathrm{a}^{-3.5}$ & $\begin{array}{l}\text { Small } \\
\text { graphite }^{c}\end{array}$ & 7 \\
\hline Fractals (Wright) & $\begin{array}{l}\text { Grains with fractal } \\
\text { dimensions (from growth) }\end{array}$ & - & - & 8 \\
\hline Sorrell & $\begin{array}{l}\text { Porous silicates, small amor. } \\
\text { carbon and silicates; small } \\
\text { graphitic particles; different } \\
\text { fractions for the diffuse ISM } \\
\text { and molecular clouds }\end{array}$ & $\begin{array}{l}\text { Discrete } \\
\text { sizes }\end{array}$ & $\begin{array}{l}\text { Small } \\
\text { graphite }^{c}\end{array}$ & 9 \\
\hline $\begin{array}{l}\text { IRAS-compatible dust } \\
\text { (Rowan-Robinson) }\end{array}$ & $\begin{array}{l}\text { Amorphous silicate and carbon } \\
\text { grains; Small graphite and } \\
\text { silicate grains }\end{array}$ & $\begin{array}{l}\text { Discrete } \\
\text { sizes }\end{array}$ & $\begin{array}{l}\text { Small } \\
\text { graphite }^{c}\end{array}$ & 10 \\
\hline $\begin{array}{l}\text { VSG model } \\
\text { (Désert et al.) }\end{array}$ & $\begin{array}{l}\text { Silicate-core mantle grains, } \\
\text { 3D VSG, 2D VSG }\end{array}$ & $\begin{array}{l}\mathrm{a}^{-3.5} \\
\mathrm{VSG}\end{array}$ & $\begin{array}{l}3 \mathrm{D} \\
\text { VSG }\end{array}$ & 11 \\
\hline $\begin{array}{l}\text { PAH model } \\
\text { (Siebenmorgen and } \\
\text { Krügel) }\end{array}$ & $\begin{array}{l}\text { Silicate and carbon grains, } \\
\text { small graphite particles, } \\
\text { PAHs }\end{array}$ & $\begin{array}{l}\mathrm{a}^{-3.5} \\
\text { PAHs }\end{array}$ & $\begin{array}{l}\text { Small } \\
\text { graphite }^{c}\end{array}$ & 12 \\
\hline
\end{tabular}

\section{Notes:}

a References: 1: Hong and Greenberg (1980); 2: Chlewicki and Greenberg (1990);

3: Chlewicki and Laureijs (1988); 4: Mathis et al. (1977); 5: Draine and Lee (1984);

6: Duley and Williams (1988), Duley et al. (1989); 7: Mathis and Whiffen (1989);

8: Wright (1987); 9: Sorrell (1992); 10: Rowan-Robinson (1992); 11: Désert et al.

(1990); 12: Siebenmorgen and Krügel (1992)

"Flat" is really an exponential-type distribution.

c "Small" graphite means that the radii of all graphite particles are $\ll 0.22 \mu \mathrm{m}$, or

$\lesssim 0.005 \mu \mathrm{m}$. Below this size, the extinction properties do not change with size. 
c.) The hydrostatic core continues to grow in size primarily due to accretion from the disk as angular momentum in the disk is transported outwards. This phase is associated with molecular outflows (presumably driven by disk accretion) and sometimes with optical jets.

d.) The disk is dissipated, the outflow dies out and the result is a pre-main sequence object $\left(M \leq 3 \mathrm{M}_{\odot}\right)$ or hydrogen-burning star $\left(M \gtrsim 10 \mathrm{M}_{\odot}\right.$; e.g. Yorke 1985), possibly surrounded by a planetary system.

Phases (a), (c) and (d) are often observed, but there is no clear-cut candidate for an object in phase (b), i.e. a protostar highly embedded in a dusty envelope which is not associated with an outflow.

As material falls into the central regions dust grains can be destroyed due to a variety of effects (direct sublimation, photon or particle sputtering, passage of grains through a shock front, etc.), which causes a dramatic decrease of the opacity. This is due to the extremely large differences between the dust and gas opacity for temperatures in the range from 1000 to $2000 \mathrm{~K}$. This effect can cause a number of instabilities in the accretion process. In spherically symmetric numerical calculations by Yorke and Krügel (1977) oscillations occurred in the protostellar envelope for cases $M_{\text {cloud }}=50 \mathrm{M}_{\odot}$ and $100 \mathrm{M}_{\odot}$ with periods $P \approx\left(r_{s u b}^{3} / G M\right)^{1 / 2}$, where $r_{s u b}$ is the radius of destruction of a major dust component.

Yorke (1988) derived approximate formulae for the destruction radii of refractory particles $\left(T_{s u b} \approx 1800 \mathrm{~K}\right)$ :

$$
r_{R} \approx 2.5 \times 10^{13} \mathrm{~cm}\left[\frac{Q_{\mathrm{eff}} L}{10^{3} \mathrm{~L}_{\odot}}\right]^{1 / 2}
$$

and for the destruction of volatile (icy) coatings $\left(T_{s u b} \approx 100 \mathrm{~K}\right)$ :

$$
r_{V} \approx 3.4 \times 10^{16} \mathrm{~cm}\left[\frac{Q_{\mathrm{eff}} L}{10^{3} \mathrm{~L}_{\odot}}\right]^{1 / 2}
$$

where $L$ is the luminosity of the central source and $Q_{\text {eff }}$ is the effective absorption cross section of a spherical dust particle normalized by the geometric cross section. When the dust "sees" the optical photons from the central source directly then $Q_{\text {eff }} \approx 1$. For the volatile component $Q_{\text {eff }}$ can vary between $Q_{\text {eff }} \approx 1$ (optically thin case) and $Q_{\text {eff }} \approx 10^{-3}$ (optically thick). Note that these approximate formulae do not include the effect of the temperature fluctuations to be expected in very small grains (see Figure 2).

An analytical study by Wuchterl (1990) predicts the generation of local hydrodynamic instabilities for protostars of all masses if opacity gradients with temperature and/or density occur. However, it is not clear if the conditions derived by Wuchterl are not only necessary but also sufficient for large scale instabilities to develop. Recently, Balluch (1991) found in his hydrodynamical calculations of low mass protostars large scale instabilities to occur simultaneously, but with unspecified causal connections, with dust disappearance. In contrast to the Yorke and Krügel (1977) high mass protostellar models, Balluch 
allowed dust to reappear spontaneously with the same tabulated optical properties when permitted by local conditions. Numerically, the instability that causes the oscillations is most likely the result of the sudden and large changes of the Rosseland mean opacities used. This may be even a purely numerical effect if the opacity is part of the grid equation.

In the paper by Balluch (1991) no physical treatment of the dust destruction and production is given. Extremely large negative opacity gradients appear abruptly as soon as one exceeds the largest temperature at which the dust opacity is tabulated. The question remains how the opacity behaves in the destruction/production zone and how steep the gradient is. What is the rôle of non-equilibrium molecular chemistry and its contribution to the opacity in the dust-free zone? How do large scale oscillations affect the dust evolution? Lenzuni et al. (1993) made an attempt of a more realistic modelling of the dust destruction in protostellar cores and derived temperature dependent Rosseland mean opacities for this case. Dust production was not considered. Obviously, this is an XNLTE problem, which can only be solved by detailed numerical modelling.

The problem is further complicated by the coagulation and fragmentation of dusty grains in the immediate vicinity of protostars and young main sequence stars. At the relatively high densities of protostellar disks dust grains can coagulate if relative velocities are smaller than $10^{2} \mathrm{~cm} \mathrm{~s}^{-1}$. At much higher relative velocities the collisions would lead to a fragmentation of the grains. Both processes can change the grain distribution and therefore the dust opacities. The coagulation process leads to more open and fluffy structures which introduces a porousity parameter in addition to the size parameter for the description of the dust density distribution. The formation of fluffy "dirty-ice" grains results in increased far-infrared and submillimeter dust opacities (e.g. Preibisch et al. 1993). Specific estimates for the value of the opacity depend on the adopted grain model, especially on the silicate-to-carbon ratio and the abundance of grains with large refractive indices (Blum 1993).

As an example of the density, temperature and velocity structure to be expected from a collapsing $1 \mathrm{M}_{\odot}$ molecular clump during phase (b) accretion, the results of numerical calculations by Yorke et al. (1993) are displayed in Figure 3. Dusty material falls at almost free fall velocities until it encounters the accretion shock front (bunching of density contour lines) which surrounds the equilibrium disk. After passing through the shock front where further dust processing (adsorption, fragmentation and coagulation) can be expected, the material settles onto the disk several scale heights above the equator. One density scale height $H_{\rho}$ corresponds to crossing two contour lines at a constant radial distance $r$ from the axis. When $H_{\rho} \ll r$, one speaks of a "thin disk". For the disk displayed in Figure $3 H_{\rho} \approx 0.1 r$.

The temperature in the equilibrium disk depends only weakly on the height $z$ from the equator and is thus basically a function of radial distance only. This means that such disks will not be unstable to convection in the $z$-direction. Only when the energy generated by viscous processes in the disk is much greater that the energy absorbed from the central source, can the disk become convectively 
unstable. At first dust can be expected to sediment toward the equator, its drift velocity depends on the ratio of dust surface area to mass. Coagulation will be prevalent and the opacity in the disk and the dust's scale height will be modified.

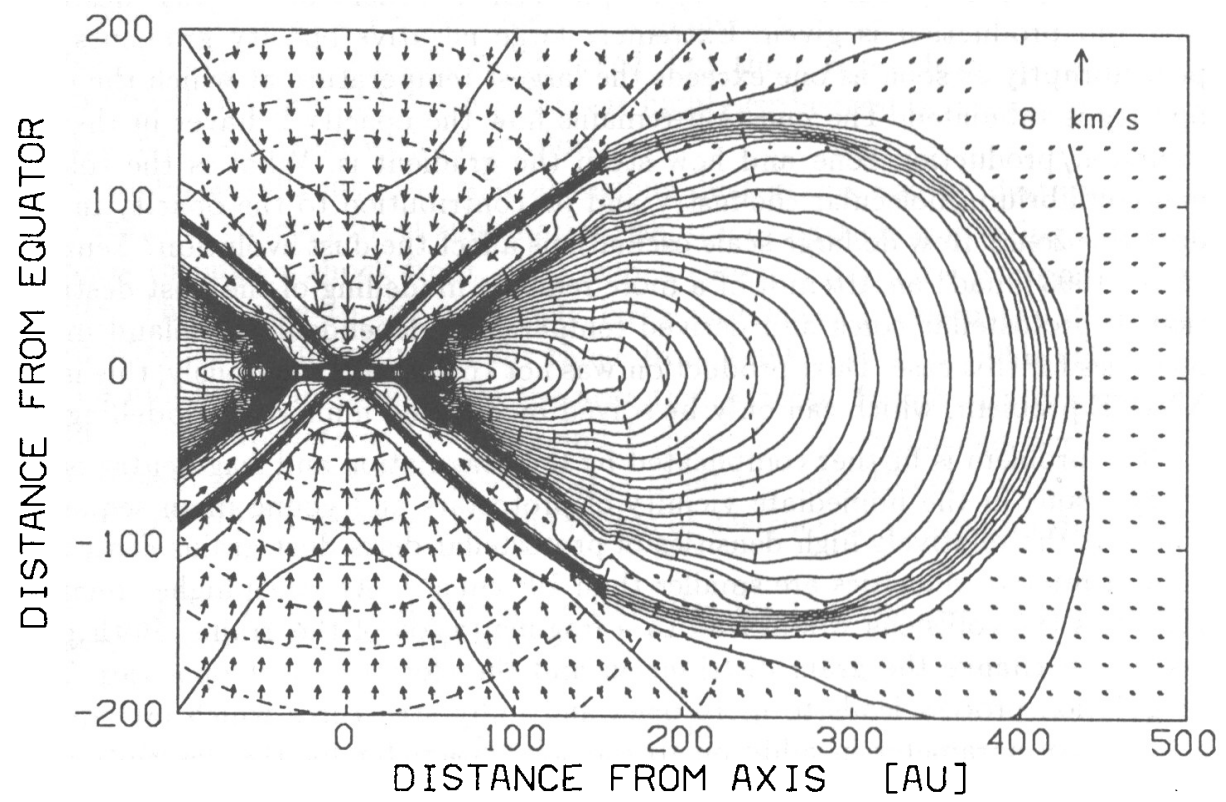

Fig. 3. Structure of temperature (dashed-dotted contour lines), density (solid contour lines) and velocity (arrows; linear scale in upper left of frame) of a protostellar disk during phase (b) at time $t=86000 \mathrm{yr}$. The outermost density (temperature) contours (in cgs units) begin at $\log \rho=-19.4(\log T=1.3)$ and adjacent contour levels are separated by $\Delta \log \rho=0.2(\Delta \log T=0.05)$.

The further evolution depends on the interplay of several poorly understood physical processes. Variations of opacity as a function of $z$ can induce convection, which will increase the outward transport of angular momentum within the disk (and the inward mass flux). Other processes may be principally responsible for angular momentum transport (magnetic fields, turbulence, gravitational torques). The accretion of material via the disk onto the central source is accompanied by mass outflow along the axis. Random turbulent velocities will further enhance coagulation (Morfill 1985) and at the same time increase the dust's scale height. Untangling this XNLTE problem is necessary for understanding the formation of planets in general and our Solar System in particular. 


\section{References}

Evans A., 1990, in Physics of Classical Novae, IAU Coll. No. 122, eds. A. Cassatella, R. Viotti (Berlin: Springer), p. 253

Allamandola L.J., Tielens A.G.G.M. (eds.), 1989, Interstellar Dust, IAU Symp. No. 135, (Dordrecht: Kluwer).

Balluch M., 1991, Astron. Astrophys. 243, 205

Blum J., Henning T., Ossenkopf V., Sablotny R., Stognienko R., Thamm E., 1993, Proceedings of Fractal 93, ed. M.M. Novak (Amsterdam: N. Holland Publ.), in press

Chlewicki G., Greenberg J.M., 1990, Astrophys. J. 365, 230

Chlewicki G., Laureijs R.J., 1988, Astron. Astrophys. 207, L11

Désert F.-X., Boulanger F., Puget J.L., 1990, Astron. Astrophys. 237, 215

Draine B.T., Lee H.M., 1984, Astrophys. J. 285, 89

Duley W.W., Williams D.A., 1988, Mon. Not. Roy. Astron. Soc. 231, 969

Duley W.W., Jones A.P., Williams D.A., 1989, Mon. Not. Roy. Astron. Soc. 236, 709

Hong S.S., Greenberg J.M., 1980, Astron. Astrophys. 88, 194

Kozasa T., Hasegawa H., Nomomoto K., 1991, Astron. Astrophys. 249, 474

Levasseur-Regourd A.C., Hasegawa H. (eds.), 1991, Origin and Evolution of Interplanetary Dust, IAU Coll. No. 126, (Dordrecht: Kluwer)

Lenzuni P., Gail H.-P., Henning T., 1993, in preparation

Mathis J.S., Rumpl W., Nordsieck K.H., 1977, Astrophys. J. 217, 425 (MRN)

Mathis J.S., Whiffen G., 1989, A strophys. J. 341, 808

Mathis J.S., 1990, private communication.

Morfill G.E., 1985, in Birth and Infancy of Stars, Les Houches Summer School XLI, eds. R. Lucas, A. Omont, R. Stora, (Amsterdam: N. Holland Publ.), p. 693

Mukai T., 1990, in Evolution of Interstellar Dust and Related Topics, eds. A. Bonetti, J.M. Greenberg, S. Aiello, (Amsterdam: N. Holland Publ.), p.397

Ossenkopf V., 1993, Astron. Astrophys. Suppl., in press.

Preibisch T., Ossenkopf V., Yorke H.W., Henning T., 1993, Astron. Astrophys. , in press.

Rowan-Robinson M., 1992, Mon. Not. Roy. Astron. Soc. 258, 787

Siebenmorgen R., Krügel E., 1992, Astron. Astrophys. 259, 614

Sorrell W.,H., 1992, Mon. Not. Roy. Astron. Soc. 255, 594

Shu F.H., Adams F.C., Lizano S., 1987, Ann. Rev. Astron. Astrophys. 25, 23

Whittet D.C.B., 1993, in Dust and chemistry in astronomy, eds. T.J. Millar and D.A.

Williams, (Bristol: Adam Hilger), p. 1

Wright E.L., 1987, Astrophys. J. 320, 818

Wuchterl G., 1990, Astron. Astrophys. 238, 83

Yorke H.W., 1985, in Birth and Infancy of Stars, Les Houches Summer School XLI, eds. R. Lucas, A. Omont, R. Stora, (Amsterdam: N. Holland Publ.), p. 648

Yorke H.W., 1988, in Radiation in Moving Gaseous Media, Eighteenth Advanced Course, Swiss Society for Astrophysics and Astronomy, (Sauverny-Versoix: Geneva Obs.), p. 195

Yorke H.W., Bodenheimer P., Laughlin G., 1993, Astrophys. J. 411, 274

Yorke H.W., Krügel E. 1977, Astron. Astrophys. 54, 183 